Comment. Math. Helv. 72 (1997) 481-499

0010-2571/97/030481-19\$1.50+0.20/0
(C) 1997 Birkhäuser Verlag, Basel

Commentarii Mathematici Helvetici

\title{
Critical values of autonomous Lagrangian systems
}

\author{
Gabriel P. Paternain and Miguel Paternain*
}

\begin{abstract}
Let $M$ be a closed manifold and $L: T M \rightarrow \mathbf{R}$ a convex superlinear Lagrangian. We consider critical values of Lagrangians as defined by R. Mañé in [5]. Let $c_{u}(L)$ denote the critical value of the lift of $L$ to the universal covering of $M$ and let $c_{a}(L)$ denote the critical value of the lift of $L$ to the abelian covering of $M$. It is easy to see that in general, $c_{u}(L) \leq c_{a}(L)$. Let $c_{0}(L)$ denote the strict critical value of $L$ defined as the smallest critical value of $L-\omega$ where $\omega$ ranges among all possible closed 1-forms. We show that $c_{a}(L)=c_{0}(L)$. We also show that if there exists $k$ such that the Euler-Lagrange flow of $L$ on the energy level $k^{\prime}$ is Anosov for all $k^{\prime} \geq k$, then $k>c_{u}(L)$. Afterwards, we exhibit a Lagrangian on a compact surface of genus two which possesses Anosov energy levels with energy $k<c_{a}(L)$, thus answering in the negative a question raised by Mañé. This example also shows that the inequality $c_{u}(L) \leq c_{a}(L)$ could be strict. Moreover, by a result of M.J. Dias Carneiro [4] these Anosov energy levels do not have minimizing measures. Finally, we describe a large class of Lagrangians for which $c_{u}(L)$ is strictly bigger than the maximum of the energy restricted to the zero section of $T M$.
\end{abstract}

Mathematics Subject Classification (1991). 58F05, 58F15.

Keywords. Lagrangian systems, minimizing measures, critical values.

\section{Introduction}

Let $M^{n}$ be a closed manifold and let $L: T M \rightarrow \mathbf{R}$ be a $C^{\infty}$ Lagrangian satisfying the following hypotheses:

- Convexity. For all $x \in M$, the restriction of $L$ to $T_{x} M$ has everywhere positive definite Hessian.

- Superlinear growth. Let || || denote a Riemannian metric on $M$. Then

$$
\lim _{\|v\| \rightarrow \infty} \frac{L(x, v)}{\|v\|}=+\infty
$$

uniformly for $x \in M$. This condition is clearly independent of the choice of Riemannian metric, since $M$ is compact.

*Both authors were supported by grants from CSIC and CONICYT \# 301 
The Euler-Lagrange equation,

$$
\frac{d}{d t}\left(\frac{\partial L}{\partial v}(x, \dot{x})\right)-\frac{\partial L}{\partial x}(x, \dot{x})=0
$$

generates a smooth complete flow $\varphi_{t}: T M \rightarrow T M$ which is defined as follows. Given $(x, v) \in T M$, consider the unique solution $x: \mathbf{R} \rightarrow M$ of the Euler-Lagrange equation with initial conditions

$$
x(0)=x, \quad \dot{x}(0)=v .
$$

Now define $\varphi_{t}: T M \rightarrow T M$ by

$$
\varphi_{t}(x, v)=(x(t), \dot{x}(t)) .
$$

Recall that the energy $E: T M \rightarrow \mathbf{R}$ is defined by

$$
E(x, v)=\frac{\partial L}{\partial v}(x, v) \cdot v-L(x, v) .
$$

Since $L$ is autonomous, $E$ is a first integral of the flow $\varphi_{t}$. Observe that for all $x \in M, E$ restricted to $T_{x} M$ is a function that achives its minimum at $(x, 0)$. Let us set

$$
e=\max _{x \in M} E(x, 0)=-\min _{x \in M} L(x, 0) .
$$

For any $k>e$, the energy level $E^{-1}(k)$ is a smooth closed hypersurface of $T M$ that intersects each tangent space $T_{x} M$ in a sphere containing the origin in its interior.

One of our aims will be the study of Anosov energy levels, that is, regular energy levels on which the flow $\varphi_{t}$ is an Anosov flow. We showed in [7] that those energy levels must verify that $k>e$ and they are free of conjugate points. In the present paper we shall describe new relations between Anosov energy levels and certain critical values recently introduced by Ricardo Mañé in his unfinished manuscript [5]. We begin by summarizing Mañé's results; proofs of these results have been given by Gonzalo Contreras, Jorge Delgado and Renato Iturriaga in [3].

Recall that the action of the Lagrangian $L$ on an absolutely continuous curve $u:[a, b] \rightarrow M$ is defined by

$$
A_{L}(u)=\int_{a}^{b} L(u(t), \dot{u}(t)) d t
$$

Given two points, $x_{1}$ and $x_{2}$ in $M$, denote by $\mathcal{C}\left(x_{1}, x_{2}\right)$ the set of absolutely continuous curves $u:[0, T] \rightarrow M$, with $u(0)=x_{1}$ and $u(T)=x_{2}$. For each $k \in \mathbf{R}$ we define the action potential $\Phi_{k}: M \times M \rightarrow \mathbf{R}$ by

$$
\Phi_{k}\left(x_{1}, x_{2}\right)=\inf \left\{A_{L+k}(u): u \in \mathcal{C}\left(x_{1}, x_{2}\right)\right\} .
$$


Mañé showed [5,3] that there exists $c(L) \in \mathbf{R}$ such that

- if $k<c(L)$, then $\Phi_{k}\left(x_{1}, x_{2}\right)=-\infty$, for all $x_{1}$ and $x_{2}$;

- if $k \geq c(L)$, then $\Phi_{k}\left(x_{1}, x_{2}\right)>-\infty$ for all $x_{1}$ and $x_{2}$ and $\Phi_{k}$ is a Lipschitz function;

- if $k \geq c(L)$, then

$$
\Phi_{k}\left(x_{1}, x_{3}\right) \leq \Phi_{k}\left(x_{1}, x_{2}\right)+\Phi_{k}\left(x_{2}, x_{3}\right),
$$

for all $x_{1}, x_{2}$ and $x_{3}$ and

$$
\Phi_{k}\left(x_{1}, x_{2}\right)+\Phi_{k}\left(x_{2}, x_{1}\right) \geq 0
$$

for all $x_{1}$ and $x_{2}$;

- if $k>c(L)$, then for $x_{1} \neq x_{2}$ we have

$$
\Phi_{k}\left(x_{1}, x_{2}\right)+\Phi_{k}\left(x_{2}, x_{1}\right)>0 .
$$

Observe that in general the action potential $\Phi_{k}$ is not symmetric, however defining $d_{k}: M \times M \rightarrow \mathbf{R}$ by

$$
d_{k}\left(x_{1}, x_{2}\right)=\Phi_{k}\left(x_{1}, x_{2}\right)+\Phi_{k}\left(x_{2}, x_{1}\right),
$$

the properties above say that $d_{k}$ is a metric for $k>c(L)$ and a pseudometric for $k=c(L)$. The number $c(L)$ is called the critical value of $L$.

It is important for our purposes to indicate that the results above also hold for coverings of $M$, i.e. suppose $\widehat{M}$ is a covering of $M$ with covering projection $p$. Take the lift of the Lagrangian $L$ to $\widehat{M}$ which is given by

$$
\widehat{L}(\widehat{x}, \widehat{v})=L(p(\widehat{x}), d p(\widehat{v})) .
$$

Then we define for each $k \in \mathbf{R}$ the action potential $\widehat{\Phi}_{k}$ just as above and the results hold for $\widehat{L}$. Thus we have a critical value for $\widehat{L}$. It is immediate that

$$
c(\widehat{L}) \leq c(L) .
$$

More generally, if $M_{1}$ and $M_{2}$ are coverings of $M$ such that $M_{1}$ covers $M_{2}$, then

$$
c\left(L_{1}\right) \leq c\left(L_{2}\right),
$$

where $L_{1}$ and $L_{2}$ denote the lifts of the Lagrangian $L$ to $M_{1}$ and $M_{2}$ respectively. Also note that if $M_{1}$ is a finite covering of $M_{2}$ then

$$
c\left(L_{1}\right)=c\left(L_{2}\right) .
$$

Among all possible coverings of $M$ there are two distinguished ones; the universal covering which we shall the denote by $\widetilde{M}$, and the abelian covering which we 
shall denote by $\bar{M}$. The latter is defined as the covering of $M$ whose fundamental group is the kernel of the Hurewicz homomorphism $\pi_{1}(M) \mapsto H_{1}(M, \mathbf{R})$. When $\pi_{1}(M)$ is abelian, $\widetilde{M}$ is a finite covering of $\bar{M}$.

The universal covering of $M$ gives rise to the critical value

$$
c_{u}(L) \stackrel{\text { def }}{=} c(\widetilde{L})
$$

and the abelian covering of $M$ gives rise to the critical value

$$
c_{a}(L) \stackrel{\text { def }}{=} c(\bar{L}) .
$$

Let $\beta: H_{1}(M, \mathbf{R}) \rightarrow \mathbf{R}$ denote Mather's action function and $\alpha: H^{1}(M, \mathbf{R}) \rightarrow$ $\mathbf{R}$ its convex dual. Mañé showed (cf. Section 2) that

$$
\alpha([\omega])=c(L-\omega)
$$

for any closed 1-form $\omega$ whose cohomology class is $[\omega]$, and he defined the strict critical value of $L$ as

$$
c_{0}(L) \stackrel{\text { def }}{=} \min \left\{c(L-\omega):[\omega] \in H^{1}(M, \mathbf{R})\right\} .
$$

We shall prove in Section 2 the following theorem.

\section{Theorem 1.1.}

$$
c_{a}(L)=c_{0}(L) .
$$

From inequality (1) it follows that

$$
c_{u}(L) \leq c_{a}(L)
$$

which naturally raises the question,

Question I. Is it true that

$$
c_{u}(L)=c_{a}(L) ?
$$

Mañé posed us the following question,

Question II. If the energy level $k$ is Anosov, is it true that

$$
k>c_{a}(L) ?
$$


We shall see that the answer to both questions is negative, however we shall show in Section 3:

Theorem 1.2. If there exists $k$ such that for all $k^{\prime} \geq k$, the energy level $k^{\prime}$ is Anosov, then

$$
k>c_{u}(L) .
$$

In Section 4 we shall exhibit on a compact surface of genus two a Lagrangian of the type kinetic energy plus a magnetic field which possesses an energy level $k$ with $k<c_{a}(L)$ and such that for all $k^{\prime} \geq k$ the energy level $k^{\prime}$ is Anosov. By Theorem 1.2

$$
c_{u}(L)<k<c_{a}(L)
$$

which gives negative answers to Questions I and II.

M.J. Dias Carneiro showed [4] that if $\mu$ is a minimizing measure (cf. Section 2 ), then its support is contained in a fixed energy level $k$ with $k \geq c_{0}(L)$. Our example and Theorem 1.1 show that Anosov energy levels do not necessarily contain minimizing measures.

In [5] Mañé describes an example of the form kinetic energy plus a magnetic field and a potential for which $e<c_{0}(L)$. We shall describe in Section 5 a large class of Lagrangians verifying the sharper inequality: $e<c_{u}(L)$ (we note that the inequality $e \leq c_{u}(L)$ always holds, cf. Section 5). Actually we prove,

Theorem 1.3. Let $\theta$ be the 1-form on $M$ given by

$$
\theta_{x}(v)=\frac{\partial L}{\partial v}(x, 0)(v)
$$

If $\theta$ is closed then

$$
e=c_{0}(L)
$$

Suppose in addition that $L(x, 0)=0$ for all $x \in M$. If $e=c_{u}(L)$, then $\theta$ is closed.

Let $M$ be a closed manifold endowed with a Riemannian metric and let $\theta$ denote a smooth 1-form on $M$. Consider a Lagrangian of the type kinetic energy plus a magnetic field, i.e.,

$$
L(x, v)=\frac{1}{2}\langle v, v\rangle_{x}+\theta_{x}(v) .
$$

The energy function associated with $L$ is

$$
E(x, v)=\frac{1}{2}\langle v, v\rangle_{x},
$$

therefore in this case $e=\max _{x \in M} E(x, 0)=0$. If $\theta$ is not closed, Theorem 1.3 immediately implies that $c_{u}(L)>0$. 
Finally let us describe one more consequence of Theorem 1.2. Suppose that the geodesic flow associated with the Riemannian metric is Anosov. Then by structural stability the Euler-Lagrange flow of $L$ is Anosov for any sufficiently large value of the energy. However, Theorem 1.2 shows that it cannot be Anosov for all values of the energy otherwise $c_{u}(L)=0$. Let $\mathcal{E}$ denote the smallest possible value of the energy such that for all $k^{\prime}>\mathcal{E}$ the energy level $k^{\prime}$ is Anosov. Theorem 1.2 immediately implies the following lower bound for $\mathcal{E}$.

\section{Corollary 1.4.}

$$
\mathcal{E} \geq c_{u}(L)
$$

In [8] we obtained lower bounds for $\mathcal{E}$ in terms of $d \theta$ and the curvature tensor of $M$ and we proved through different methods that $\mathcal{E}$ cannot vanish if $\theta$ is not closed.

We would like to thank Gonzalo Contreras and Renato Iturriaga for many useful discussions and for making their manuscript [3] readily available to us.

\section{Proof of Theorem 1.1}

We begin by recalling the main ingredients of Mather's theory [6]; results on minimal geodesics on arbitrary Riemannian manifolds were previously obtained by V. Bangert [1].

Let $\mathcal{M}(L)$ be the set of probabilities on the Borel $\sigma$-algebra of $T M$ that have compact support and are invariant under the flow $\varphi_{t}$. Let $H_{1}(M, \mathbf{R})$ be the first real homology group of $M$. Given a closed 1-form $\omega$ on $M$ and $\rho \in H_{1}(M, \mathbf{R})$, let $\langle\omega, \rho\rangle$ denote the integral of $\omega$ on any closed curve in the homology class $\rho$. If $\mu \in \mathcal{M}(L)$, its homology is defined as the unique $\rho(\mu) \in H_{1}(M, \mathbf{R})$ such that

$$
<\omega, \rho(\mu)>=\int \omega d \mu
$$

for all closed 1-forms on $M$. The integral on the right-hand side is with respect to $\mu$ with $\omega$ considered as a function $\omega: T M \rightarrow \mathbf{R}$. The function $\rho: \mathcal{M}(L) \rightarrow$ $H_{1}(M, \mathbf{R})$ is surjective [6].

The action of $\mu \in \mathcal{M}(L)$ is defined by

$$
A_{L}(\mu)=\int L d \mu
$$

Finally we define the function $\beta: H_{1}(M, \mathbf{R}) \rightarrow \mathbf{R}$ by

$$
\beta(\gamma)=\inf \left\{A_{L}(\mu): \rho(\mu)=\gamma\right\}
$$


The function $\beta$ is convex and superlinear and the infimum can be shown to be a minimum [6] and the measures at which the minimum is attained are called minimizing measures. In other words, $\mu \in \mathcal{M}(L)$ is a minimizing measure if

$$
\beta(\rho(\mu))=A_{L}(\mu)
$$

An absolutely continuous curve $u:[0, T] \rightarrow \bar{M}$ is called an $\bar{L}$-minimizer if it minimizes the action $A_{\bar{L}}$ over the class of absolutely continuous curves defined on $[0, T]$ with the same end points as $u$.

Choose a basis $\gamma_{1}, \ldots, \gamma_{r}$ of $H_{1}(M, \mathbf{R})$ and let $\omega_{1}, \ldots, \omega_{r}$ be closed 1-forms whose cohomology classes form a dual basis in $H^{1}(M, \mathbf{R})$. Given two points $\bar{x}$ and $\bar{y}$ in $\bar{M}$, their difference vector $\bar{x}-\bar{y} \in H_{1}(M, \mathbf{R})$ is defined by

$$
\bar{x}-\bar{y}=\sum_{i=1}^{r}\left(\int_{\tau} \bar{\omega}_{i}\right) \gamma_{i},
$$

where $\tau$ is a $C^{1}$ curve in $\bar{M}$ connecting $\bar{y}$ to $\bar{x}$ and $\bar{\omega}_{i}$ is the lift of $\omega_{i}$ to $\bar{M}$.

We shall need the following proposition due to Mather [6, Proposition 1].

Proposition 2.1. Let $u_{i}:\left[0, T_{i}\right] \rightarrow \bar{M}$ be a sequence of $\bar{L}$-minimizers such that $T_{i} \rightarrow \infty$ and $\frac{u_{i}\left(T_{i}\right)-u_{i}(0)}{T_{i}} \rightarrow \gamma \in H_{1}(M, \mathbf{R})$ as $i \rightarrow \infty$. Then

$$
\lim _{i \rightarrow \infty} \frac{1}{T_{i}} A_{\bar{L}}\left(u_{i}\right)=\beta(\gamma)
$$

Mañé $[5,3]$ established a connection between the critical values of a Lagrangian as described in the introduction and the convex dual of Mather's $\beta$ function. He showed that

$$
c(L)=-\min \left\{\int L d \mu: \mu \in \mathcal{M}(L)\right\}
$$

Let us recall how the convex dual $\alpha: H^{1}(M, \mathbf{R}) \rightarrow \mathbf{R}$ of $\beta$ is defined. Since $\beta$ is convex and superlinear we can set

$$
\alpha([\omega])=\max \left\{<\omega, \gamma>-\beta(\gamma): \gamma \in H_{1}(M, \mathbf{R})\right\},
$$

where $\omega$ is any closed 1 -form whose cohomology class is $[\omega]$. The function $\alpha$ is also convex and superlinear. Mather [6] showed that

$$
\alpha([\omega])=-\min \left\{\int(L-\omega) d \mu: \mu \in \mathcal{M}(L)\right\},
$$


and therefore using (3) we obtain the remarkable equality

$$
c(L-\omega)=\alpha([\omega])
$$

for any closed 1-form $\omega$ whose cohomology class is $[\omega]$. From the duality bewteen $\alpha$ and $\beta$ we have

$$
\begin{gathered}
-\beta(0)=\min \left\{\alpha([\omega]):[\omega] \in H^{1}(M, \mathbf{R})\right\} \\
=\min \left\{c(L-\omega):[\omega] \in H^{1}(M, \mathbf{R})\right\} .
\end{gathered}
$$

Finally, Mañé defined the strict critical value of $L$ as

$$
c_{0}(L) \stackrel{\text { def }}{=} \min \left\{c(L-\omega):[\omega] \in H^{1}(M, \mathbf{R})\right\} .
$$

We shall use the following result of Mañé $[5,3]$ that exhibits the relevance of the critical values for variational problems on fixed energy levels.

Theorem 2.2. Suppose $k>c(L)$. Then, given $x_{1} \neq x_{2}$ in $M$, there exists a solution $x(t)$ of the Euler-Lagrange equation with energy $k$ such that for some $T>0, x(0)=x_{1}, x(T)=x_{2}$ and

$$
A_{L+k}\left(\left.x\right|_{[0, T]}\right)=\Phi_{k}\left(x_{1}, x_{2}\right) .
$$

Theorem 2.2 also holds for coverings, i.e. if we replace $M$ by a covering and $L$ by the lifted Lagrangian.

We state now the result of M.J. Dias Carneiro [4] that we mentioned in the introduction.

Theorem 2.3. If $\mu$ is a minimizing measure with homology $\gamma$, then its support is contained in a fixed energy level $k$ and $k=\alpha([\omega])$, where $[\omega]$ is the slope of a supporting hyperplane through $(\gamma, \beta(\gamma))$. In particular, $k \geq c_{0}(L)$.

We now show,

Lemma 2.4. For any closed 1-form $\omega$ on $M$ we have

$$
c_{a}(L-\omega)=c_{a}(L) .
$$

Proof Let $\bar{\omega}$ denote the lift of $\omega$ to the abelian covering of $M$. Since the form $\bar{\omega}$ is exact, the action potential of $\bar{L}-\bar{\omega}$ and the action potential of $\bar{L}$ coincide on the diagonal of $\bar{M} \times \bar{M}$. This readily implies the lemma. 
Proof of Theorem 1.1. By equality (4) we can take a closed 1-form $\omega$ such that

$$
c_{0}(L)=c(L-\omega)
$$

By the previous lemma and (1) we have

$$
c_{a}(L)=c_{a}(L-\omega) \leq c(L-\omega)=c_{0}(L)
$$

To complete the proof of the theorem we need to show

$$
c_{a}(L) \geq c_{0}(L)
$$

We shall assume that $\bar{M}$ is non-compact otherwise $\bar{M}$ is a finite covering of $M$ and by $(2), c_{a}(L)=c(L) \geq c_{0}(L)$.

Suppose that $c_{a}(L)<c_{0}(L)$. Take $k$ such that $c_{a}(L)<k<c_{0}(L)$. Fix a point $\bar{q} \in \bar{M}$ and take a sequence of points $\bar{q}_{i}$ such that $d\left(\bar{q}, \bar{q}_{i}\right) \rightarrow \infty$ (we provide $M$ with a Riemannian metric and we lift it to $\bar{M}$ ).

By Theorem 2.2 there exists for each $i$ a solution $\bar{x}_{i}(t)$ of the Euler-Lagrange equations with energy $k$ such that for some $T_{i}>0, \bar{x}_{i}(0)=\bar{q}, \bar{x}_{i}\left(T_{i}\right)=\bar{q}_{i}$ and

$$
A_{\bar{L}+k}\left(\left.\bar{x}_{i}\right|_{\left[0, T_{i}\right]}\right)=\bar{\Phi}_{k}\left(\bar{q}, \bar{q}_{i}\right)
$$

Since the solutions have energy $k$, there exists a constant $a$ such that $\left\|\dot{\bar{x}}_{i}(t)\right\|<a$ for all $i$ and all $t$. Therefore

$$
d\left(\bar{q}, \bar{q}_{i}\right) \leq a T_{i} .
$$

It follows that $T_{i} \rightarrow \infty$. Let $\mu_{i}$ denote the probability measure uniformly distributed along the projection of $\left.\bar{x}_{i}\right|_{\left[0, T_{i}\right]}$ to $M$ and let $\mu$ denote a point of accumulation of $\mu_{i}$. Equality (6) implies that $\left.\bar{x}_{i}\right|_{\left[0, T_{i}\right]}$ are $\bar{L}$-minimizers and we thus can apply Proposition 2.1 to deduce that

$$
\lim _{i \rightarrow \infty} \frac{1}{T_{i}} A_{\bar{L}}\left(\left.\bar{x}_{i}\right|_{\left[0, T_{i}\right]}\right)=A_{\bar{L}}(\mu)=\beta(\rho(\mu)) .
$$

Therefore $\mu$ is a minimizing measure. Since the support of $\mu$ is clearly contained in the energy level $k$, Theorem 2.3 implies that $k \geq c_{0}(L)$ thus obtaining a contradiction. 


\section{Proof of Theorem 1.2}

The theorem is an immediate consequence of the following two propositions and the fact that the Anosov energy levels form an open set.

Proposition 3.1. If $k<c_{u}(L)$, there exists a solution $\widetilde{x}: \mathbf{R} \rightarrow \widetilde{M}$ of the EulerLagrange equation with energy $k^{\prime} \geq k$ and $T>0$ such that $\widetilde{x}(0)=\widetilde{x}(T)$.

Proposition 3.2. If the energy level $k^{\prime}$ is Anosov, then any solution $\widetilde{x}: \mathbf{R} \rightarrow \widetilde{M}$ of the Euler-Lagrange equation with energy $k^{\prime}$ is one to one.

Proof of Proposition 3.1. By the definition of the critical value, if $k<c_{u}(L)$ there exist $T_{0}>0$ and an absolutely continuous closed curve $u:\left[0, T_{0}\right] \rightarrow \widetilde{M}$ with $\widetilde{p} \stackrel{\text { def }}{=} u(0)=u\left(T_{0}\right)$ such that

$$
A_{\widetilde{L}+k}(u)<0 .
$$

Let $\mathcal{C}\left(T_{0}\right)$ denote the set of all absolutely continuous closed curves $w:[0, t] \rightarrow$ $\widetilde{M}$ such that $t \leq T_{0}$ and $\widetilde{p}=w(0)=w(t)$. The same arguments that prove Tonelli's Theorem [6] allow us to conclude that the action $A_{\widetilde{L}+k}$ takes a finite minimum value on the set $\mathcal{C}\left(T_{0}\right)$ and minimizers are solutions of the Euler-Lagrange equation. In other words, there exists a solution $\widetilde{x}: \mathbf{R} \rightarrow \widetilde{M}$ of the Euler-Lagrange equation and $T \in\left[0, T_{0}\right]$ such that $\left.\widetilde{x}\right|_{[0, T]}$ minimizes the action $A_{\widetilde{L}+k}$ on the set $\mathcal{C}\left(T_{0}\right)$. By (7) the minimum value has to be negative and therefore $T \neq 0$. Let $k^{\prime}$ be the energy of the solution $\widetilde{x}$. To complete the proof of the proposition we need to show that $k^{\prime} \geq k$.

Let us define for each $\lambda>0$ the following function:

$$
F(\lambda) \stackrel{\text { def }}{=} \int_{0}^{\lambda T}(\widetilde{L}+k)\left(\widetilde{x}_{\lambda}, \dot{\widetilde{x}}_{\lambda}\right) d t
$$

where $\widetilde{x}_{\lambda}(t):[0, \lambda T] \rightarrow \widetilde{M}$ is defined as $\widetilde{x}_{\lambda}(t)=\widetilde{x}\left(\frac{t}{\lambda}\right)$. Let us compute $F^{\prime}(1)$.

$$
F^{\prime}(\lambda)=T \widetilde{L}\left(\widetilde{x}_{\lambda}(\lambda T), \dot{\tilde{x}}_{\lambda}(\lambda T)\right)+\int_{0}^{\lambda T} \frac{\partial \widetilde{L}}{\partial \lambda} d t+k T
$$

but

$$
\begin{aligned}
\frac{\partial \widetilde{L}}{\partial \lambda} & =\frac{\partial \widetilde{L}}{\partial \widetilde{x}} \frac{\partial \widetilde{x}_{\lambda}}{\partial \lambda}+\frac{\partial \widetilde{L}}{\partial v} \frac{\partial \dot{\widetilde{x}}_{\lambda}}{\partial \lambda} \\
& =-\frac{\partial \widetilde{L}}{\partial \widetilde{x}} \dot{\widetilde{x}}_{\lambda}\left(\frac{t}{\lambda}\right) \frac{t}{\lambda^{2}}-\frac{\partial \widetilde{L}}{\partial v}\left(\frac{1}{\lambda^{3}} \ddot{\widetilde{x}}_{\lambda}\left(\frac{t}{\lambda}\right) t+\frac{\dot{\widetilde{x}}_{\lambda}\left(\frac{t}{\lambda}\right)}{\lambda^{2}}\right)
\end{aligned}
$$


therefore

$$
\begin{aligned}
F^{\prime}(1) & =T \widetilde{L}(\widetilde{x}(T), \dot{\tilde{x}}(T))+T k-\int_{0}^{T}\left(\frac{\partial \widetilde{L}}{\partial \widetilde{x}} \dot{\widetilde{x}}+\frac{\partial \widetilde{L}}{\partial v} \ddot{\widetilde{x}}\right) t d t-\int_{0}^{T} \frac{\partial \widetilde{L}}{\partial v} \dot{\tilde{x}} d t \\
& =T \widetilde{L}(\widetilde{x}(T), \dot{\widetilde{x}}(T))+T k-\int_{0}^{T} \frac{\partial \widetilde{L}}{\partial v} \dot{\tilde{x}} d t-\int_{0}^{T} \frac{d}{d t}(\widetilde{L}) t d t \\
& =T \widetilde{L}(\widetilde{x}(T), \dot{\widetilde{x}}(T))+T k-\int_{0}^{T} \frac{\partial \widetilde{L}}{\partial v} \dot{\widetilde{x}} d t+\int_{0}^{T} \widetilde{L} d t-\left.\widetilde{L} t\right|_{0} ^{T} \\
& =T k-\int_{0}^{T} k^{\prime} d t \\
& =T\left(k-k^{\prime}\right) .
\end{aligned}
$$

Now observe that since $\left.\widetilde{x}\right|_{[0, T]}$ minimizes the action $A_{\widetilde{L}+k}$ on the set $\mathcal{C}\left(T_{0}\right)$ we must have $F^{\prime}(1) \leq 0$ and therefore $k^{\prime} \geq k$ as desired.

Remark 3.3. Note that the proposition also holds if we consider any covering of $M$ and its associated critical value and not just the universal covering.

Also observe that if in the previous proof $T \neq T_{0}$ then by the minimizing condition $F^{\prime}(1)$ is actually zero and therefore the energy of $\widetilde{x}$ must be precisely $k$. If this were the case we could have had the same conclusion of Theorem $1.2 \mathrm{but}$ only assuming that the energy level $k$ is Anosov.

Proof of Proposition 3.2. A crucial ingredient in the proof of this proposition are the results we obtained in [7]. Let us recall them. Let $\pi: T M \rightarrow M$ denote the canonical projection and, if $v \in T M$, let $V(v)$ denote the vertical fibre at $v$ defined as usual as the kernel of $d \pi_{v}: T_{v} T M \rightarrow T_{\pi(v)} M$. If the energy level $\Sigma \stackrel{\text { def }}{=} E^{-1}\left(k^{\prime}\right)$ is Anosov, let $E^{s}$ denote the weak stable subbundle and let $\mathcal{W}^{s}$ denote the weak stable foliation. We showed in [7] that for all $v$ in the energy level $k^{\prime}$ we have

$$
E^{s}(v) \cap V(v)=\{0\} .
$$

This is equivalent to saying that the weak stable foliation $\mathcal{W}^{s}$ is transverse to the fibres of the fibration by $(n-1)$-spheres

$$
\left.\pi\right|_{\Sigma}: \Sigma \rightarrow M
$$

Let $\widetilde{M}$ denote the universal covering of $M$ with projection $p: \widetilde{M} \rightarrow M$. Let $\underset{\widetilde{\Sigma}}{\widetilde{\Sigma}}$ denote the lifting of $\Sigma$ to $T \widetilde{M}$ via the map $d p: T \widetilde{M} \rightarrow T M$. Observe that $\widetilde{\Sigma}$ coincides with the energy level $k^{\prime}$ of the lifted Lagrangian $\widetilde{L}$. We also have a fibration by $(n-1)$-spheres

$$
\left.\widetilde{\pi}\right|_{\widetilde{\Sigma}}: \widetilde{\Sigma} \rightarrow \widetilde{M} .
$$


Let $\widetilde{\mathcal{W}^{s}}$ be the lifted foliation which is in turn a weak stable foliation for the Euler-Lagrange flow of $\widetilde{L}$ restricted to $\widetilde{\Sigma}$. The foliation $\widetilde{\mathcal{W}}^{s}$ is also transverse to the fibration $\left.\widetilde{\pi}\right|_{\widetilde{\Sigma}}: \widetilde{\Sigma} \rightarrow \widetilde{M}$ since the map $d p$ is a local diffeomorphism. Since the fibres are compact a result of Ehresman (cf. [2]) implies that for every $v \in \widetilde{\Sigma}$ the map

$$
\left.\widetilde{\pi}\right|_{\widetilde{\mathcal{W}}^{s}(v)}: \widetilde{\mathcal{W}}^{s}(v) \rightarrow \widetilde{M}
$$

is a covering map. Since $\widetilde{M}$ is simply connected, $\left.\widetilde{\pi}\right|_{\widetilde{\mathcal{W}}^{s}(v)}$ is in fact a diffeomorphism and $\widetilde{\mathcal{W}^{s}}(v)$ is simply connected. Consequently, $\widetilde{\mathcal{W}^{s}}(v)$ intersects each fibre of the fibration $\left.\widetilde{\pi}\right|_{\widetilde{\Sigma}}: \widetilde{\Sigma} \rightarrow \widetilde{M}$ at just one point.

Now let $\widetilde{x}: \mathbf{R} \rightarrow \widetilde{M}$ be a solution of the Euler-Lagrange equation with energy $k^{\prime}$. Then for each $t \in \mathbf{R},(\widetilde{x}(t), \dot{\widetilde{x}}(t))$ belongs to $\widehat{\mathcal{W}}^{s}(\widetilde{x}(0), \dot{\widetilde{x}}(0))$ and therefore $\widetilde{x}(0)=\widetilde{x}(T)$ for $T \neq 0$ if and only if $\widetilde{x}$ gives rise to a closed orbit of the EulerLagrange flow of $\widetilde{L}$. But $\widetilde{\mathcal{W}^{s}}(\widetilde{x}(0), \dot{\widetilde{x}}(0))$ cannot contain periodic orbits since it is simply connected, and we conclude that $\widetilde{x}: \mathbf{R} \rightarrow \widetilde{M}$ is one to one.

\section{The example}

In this section we shall exhibit a convex superlinear Lagrangian $L$ on a closed orientable surface of genus two with energy levels $k^{\prime}$ which are Anosov for all $k^{\prime} \geq 1 / 2$ and $1 / 2<c_{0}(L)$. By Theorem $1.2, c_{u}(L)<1 / 2$ and we obtain negative answers to Questions I and II in the introduction.

We start with a few preliminaries. Let $M$ be a closed manifold endowed with a Riemannian metric and let $\theta$ denote a smooth 1-form on $M$. Our Lagrangian will be of the form

$$
L(x, v)=\frac{1}{2}\langle v, v\rangle_{x}-\theta_{x}(v)
$$

The energy function associated with $L$ is

$$
E(x, v)=\frac{1}{2}\langle v, v\rangle_{x},
$$

therefore in this case $e=\max _{x \in M} E(x, 0)=0$. Let $\mathcal{L}: T M \rightarrow T^{*} M$ denote the Legendre transform associated with $L$ and let $\omega_{\text {can }}$ denote the canonical symplectic form of $T^{*} M$. It is well known that the Euler-Lagrange flow $\varphi_{t}$ associated with $L$ can be obtained as the Hamiltonian flow of $E$ with respect to the symplectic form $\mathcal{L}^{*} \omega_{\text {can }}$.

Let $\pi: T M \rightarrow M$ denote the canonical projection. Let $\omega_{0}$ denote the symplectic form on $T M$ obtained by pulling back $\omega_{\text {can }}$ via the Riemannian metric. An easy computation shows that

$$
\mathcal{L}^{*} \omega_{\text {can }}=\omega_{0}+\pi^{*} d \theta
$$


The flow $\varphi_{t}$ models the motion of a particle of unit mass and charge under the effect of a magnetic field, whose Lorentz force $Y: T M \rightarrow T M$ is the bundle map determined uniquely by:

$$
d \theta_{p}(u, v)=\left\langle Y_{p}(u), v\right\rangle,
$$

for all $u$ and $v$ in $T M$.

We shall need the Jacobi equation associated with the Euler-Lagrange flow of $L$, this equation was obtained in [8] but we include its derivation here for completeness. Take a curve $Z:(-\epsilon, \epsilon) \rightarrow T M$ with $Z(0)=v, Z^{\prime}(0)=\xi$ and consider the variation $f(s, t)=\pi\left(\varphi_{t}(Z(s))\right)$. Set $J_{\xi}(t) \stackrel{\text { def }}{=} \frac{\partial f}{\partial s}(0, t), \gamma_{s} \stackrel{\text { def }}{=} f(s, t)$ and $\gamma_{0} \stackrel{\text { def }}{=} \gamma$. Let $R$ denote the Riemann curvature tensor of the Riemannian metric and let $D$ denote covariant derivative. Recall the well known identity:

$$
\frac{D}{d s} \frac{D}{d t} \frac{\partial f}{\partial t}=\frac{D}{d t} \frac{D}{d t} \frac{\partial f}{\partial s}+R\left(\frac{\partial f}{\partial t}, \frac{\partial f}{\partial s}\right) \frac{\partial f}{\partial t} .
$$

Since the Euler-Lagrange flow $\varphi_{t}$ can be obtained as the Hamiltonian flow of $E$ with respect to the symplectic form $\mathcal{L}^{*} \omega_{c a n}$, it can be easily verified using (9) that $\gamma_{s}$ satisfies the following equation of motion (Newton's law):

$$
\frac{D}{d t} \dot{\gamma_{s}}=Y\left(\dot{\gamma}_{s}\right)
$$

Combining the last two equalities we obtain:

$$
\ddot{J}_{\xi}+R\left(\dot{\gamma}, J_{\xi}\right) \dot{\gamma}=\frac{D}{d s}\left(Y\left(\dot{\gamma}_{s}\right)\right)
$$

Note that the map $(p, v) \rightarrow Y(p, v)$ is a $(1,1)$-tensor. Thus using the covariant derivative $\nabla$ on $(1,1)$-tensors induced by the Riemannian connection we obtain:

$$
\frac{D}{d s} Y\left(\dot{\gamma}_{s}\right)=\left(\nabla_{J_{\xi}} Y\right)\left(\dot{\gamma}_{s}\right)+Y\left(\dot{J}_{\xi}\right)
$$

and we deduce the Jacobi equation:

$$
\ddot{J}_{\xi}+R\left(\dot{\gamma}, J_{\xi}\right) \dot{\gamma}-Y\left(\dot{J}_{\xi}\right)-\left(\nabla_{J_{\xi}} Y\right)(\dot{\gamma})=0 .
$$

Suppose now that $M$ is a closed oriented surface and let $\Omega_{a}$ denote the area form associated with the Riemannian metric. Any other 2 -form $\Omega$ can be written as $\Omega=F \Omega_{a}$ for a smooth function $F: M \rightarrow \mathbf{R}$. The form $\Omega$ is exact if and only if

$$
\int_{M} F \Omega_{a}=0
$$

We shall exhibit now a Riemannian metric on an orientable surface of genus two and a smooth function $F: M \rightarrow \mathbf{R}$ such that $F \Omega_{a}$ is exact and if we write 
$F \Omega_{a}=d \theta$, then the Euler-Lagrange flow of the Lagrangian given in (8) is Anosov on the energy level $k^{\prime}$ for all $k^{\prime} \geq 1 / 2$ and $1 / 2<c_{0}(L)$.

To construct the Riemannian metric and the function $F$ we proceed as follows. Let $S_{l}$ denote the one-parameter family of negatively curved compact surfaces of genus two that is indicated in Figure 1, where $l$ is the length of the unit speed closed geodesic $\gamma$. Let $K_{l}$ denote the Gaussian curvature of $S_{l}$. The curve $\gamma$ divides $S_{l}$ into two surfaces with boundary: $S_{l}^{+}$and $S_{l}^{-}$. We shall assume that for each $l, S_{l}$ admits an isometric involution $I$ that fixes $\gamma$ and interchanges $S_{l}^{+}$ with $S_{l}^{-}$. Morever, we suppose that there exists a disk $D^{+}$contained in $S_{l}^{+}$such that the metric on it does not change with $l$ and it has constant curvature -1 . Let $D^{-} \stackrel{\text { def }}{=} I\left(D^{+}\right)$. We choose the orientation of $\gamma$ induced by $S_{l}^{+}$.

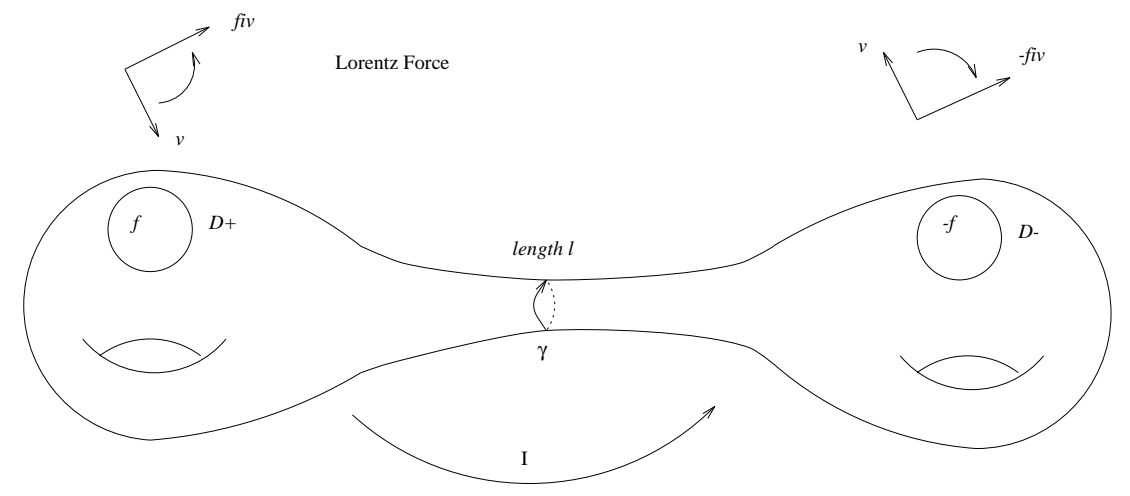

Figure 1. The surface $S_{l}$ and the magnetic field.

Let $f: S_{l}^{+} \rightarrow \mathbf{R}$ be a non-negative function with support contained in $D^{+}$and such that

$$
-1+f^{2}(x) \pm\langle\nabla f(x), i v\rangle<0
$$

for all $(x, v)$ in the unit sphere bundle of $D^{+} ; i v$ denotes the vector $v$ rotated $\pi / 2$ according to the orientation of $S_{l}$. Set $m=\int_{D^{+}} f \Omega_{a}>0$ and note that $m$ is independent of $l$. Finally our function $F$ will be defined as

$$
F(x)= \begin{cases}f(x) & \text { if } x \in S_{l}^{+} \\ -f(I x) & \text { if } x \in S_{l}^{-}\end{cases}
$$

Clearly

$$
\int_{S_{l}} F \Omega_{a}=0
$$

Let $L_{l}$ denote the Lagrangian given by the metric on $S_{l}$ and a 1-form $\theta$ that satisfies $d \theta=F \Omega_{a}$. Note that we can express the Lorentz force associated to the magnetic field $d \theta$ as

$$
Y(v)=F(\pi(v)) i v
$$


Let us compute the action of $L_{l}+c_{0}\left(L_{l}\right)$ on $\gamma$.

$$
A_{L_{l}+c_{0}\left(L_{l}\right)}(\gamma)=\frac{l}{2}+c_{0}\left(L_{l}\right) l-\int_{\gamma} \theta
$$

By Stokes Theorem,

$$
A_{L_{l}+c_{0}\left(L_{l}\right)}(\gamma)=l\left(\frac{1}{2}+c_{0}\left(L_{l}\right)\right)-\int_{D^{+}} f \Omega_{a}=l\left(\frac{1}{2}+c_{0}\left(L_{l}\right)\right)-m .
$$

Observe that $\gamma$ is null-homologous and since by Theorem 1.1, $c_{0}\left(L_{l}\right)=c_{a}\left(L_{l}\right)$, it follows from the definition of critical value that

$$
A_{L_{l}+c_{0}\left(L_{l}\right)}(\gamma) \geq 0
$$

therefore

$$
l\left(\frac{1}{2}+c_{0}\left(L_{l}\right)\right)-m \geq 0
$$

which implies, since $m$ is positive, that

$$
\lim _{l \rightarrow 0} c_{0}\left(L_{l}\right)=\infty
$$

We shall show now that for all $l$, the energy level $k^{\prime}$ for $k^{\prime} \geq 1 / 2$ is Anosov. Combining this fact with (13) it follows that for $l$ small enough we obtain a surface $S_{l}$ and a function $F$ with the desired properties.

We are going to define for each $v$ with energy $k^{\prime}$, the corresponding strong stable space $E^{s s}(v)$ and the strong unstable space $E^{s u}$. Let $c: \mathbf{R} \rightarrow S_{l}$ denote the solution of the Euler-Lagrange equation with energy $k^{\prime}>0$ and initial condition $v$. Since $\{\dot{c}, i \dot{c}\}$ is an orthonormal basis of $T_{\dot{c}} S_{l}$ we can write any Jacobi field $J$ as

$$
J=x \dot{c}+y i \dot{c} .
$$

We only need to consider Jacobi fields arising from variations in the energy level, that is $(J(0), \dot{J}(0)) \in T_{v} E^{-1}\left(k^{\prime}\right)$ which is equivalent to saying that $\langle\dot{J}, \dot{c}\rangle \equiv 0$. Since $J$ must also satisfy the Jacobi equation (10) a straightforward computation using (12) shows that the functions $x$ and $y$ must satisfy the following scalar equations:

$$
\begin{gathered}
\dot{x}=F y, \\
\ddot{y}+\left(2 k^{\prime} K_{l}(c)+F^{2}(c)-\langle\nabla F(c), i \dot{c}\rangle\right) y=0 .
\end{gathered}
$$

Now the same proof that shows that the geodesic flow on a compact surface of negative curvature is Anosov allows us to deduce that if any $v$ with energy $k^{\prime}$ satisfies

$$
2 k^{\prime} K_{l}(\pi v)+F^{2}(\pi v)-\langle\nabla F(\pi v), i v\rangle<0,
$$


then there exist constants $C, \lambda>0$, and solutions $y^{s}, y^{u}$ of the equation (15) such that

$$
\begin{gathered}
\left|y^{s}(t)\right| \leq C e^{-\lambda t}, \quad \text { for all } t \geq 0, \\
\left|y^{u}(t)\right| \leq C e^{\lambda t}, \quad \text { for all } t \leq 0 .
\end{gathered}
$$

Since $F$ is uniformly bounded, we can associate for each pair of solutions $y^{s}, y^{u}$ as above, a pair of solutions $x^{s}, x^{u}$ of equation (14) by setting

$$
\begin{gathered}
x^{s}(0) \stackrel{\text { def }}{=}-\int_{0}^{\infty} F(c(t)) y^{s}(t) d t, \\
x^{u}(0) \stackrel{\text { def }}{=} \int_{0}^{\infty} F(c(-t)) y^{u}(-t) d t .
\end{gathered}
$$

It is easily seen that

$$
\begin{aligned}
x^{s}(t) & =-\int_{t}^{\infty} F(c(\tau)) y^{s}(\tau) d \tau, \\
x^{u}(t) & =\int_{-\infty}^{t} F(c(\tau)) y^{u}(\tau) d \tau .
\end{aligned}
$$

Then $x^{s}(t)$ and $x^{u}(t)$ converge exponentially fast as $t \rightarrow \infty$. Let $J^{s}$ denote the unique Jacobi field determined by the initial conditions $\left(x^{s}(0), y^{s}(0), F(\pi v) y^{s}(0)\right.$, $\left.\dot{y}^{s}(0)\right)$ and let $J^{u}$ denote the unique Jacobi field determined by the initial conditions $\left(x^{u}(0), y^{u}(0), F(\pi v) y^{u}(0), \dot{y}^{u}(0)\right)$. Then

$$
\begin{aligned}
& E^{s s}(v)=\mathbf{R}\left(J^{s}(0), \dot{J}^{s}(0)\right), \\
& E^{s u}(v)=\mathbf{R}\left(J^{u}(0), \dot{J}^{u}(0)\right)
\end{aligned}
$$

are clearly the strong stable and unstable spaces.

Therefore if equation (16) is satisfied for all $v$ with energy $k^{\prime}$, the EulerLagrange flow on the energy level $k^{\prime}$ is Anosov. Let us check that equation (16) is satisfied for our choice of $F$.

Observe that outside $D^{+}$and $D^{-}$the function $F$ vanishes and therefore inequality (16) reduces to

$$
2 k^{\prime} K_{l}(\pi v)<0
$$

which is satisfied since we have chosen our surfaces with negative curvature. Inside the disks the metric does not change with $l$ and has constant negative curvature -1 , and thus (16) reduces to

$$
-2 k^{\prime}+F^{2}(\pi v)-\langle\nabla F(\pi v), i v\rangle<0
$$

which is satisfied for any $k^{\prime} \geq 1 / 2$ because of our choice of $f$ in (11).

Finally we note that (13) implies that the gap between $c_{u}(L)$ and $c_{0}(L)$ can be made as large as one wishes. 


\section{Proof of Theorem $\mathbf{1 . 3}$}

Let us prove first that in general

$$
e \leq c_{u}(L)
$$

Take a point $\widetilde{x} \in \widetilde{M}$ such that $e=-L(p(\widetilde{x}), 0)$. Then by considering a curve $u:[0, T] \rightarrow \widetilde{M}$ such that $u(t)=\widetilde{x}$ for all $t \in[0, T]$ we have

$$
\widetilde{\Phi}_{c_{u}(L)}(\widetilde{x}, \widetilde{x}) \leq\left(-e+c_{u}(L)\right) T
$$

which implies inequality (17).

We begin now with the proof of the theorem. Let $\psi(x) \stackrel{\text { def }}{=} L(x, 0)$ and $L_{0}(x, v)$ $\stackrel{\text { def }}{=} L(x, v)-\theta_{x}(v)-\psi(x)$. Then $L_{0}(x, 0)=L(x, 0)-\psi(x) \equiv 0$. Note that $L_{0}(x, *)$ is a convex superlinear function and $\frac{\partial L_{0}}{\partial v}(x, 0) \equiv 0$. Hence $L_{0}(x, *)$ has its minimum at $v=0$, therefore

$$
L_{0}(x, v) \geq 0
$$

and it vanishes if and only if $v=0$. Observe that

$$
e=-\min _{x \in M} L(x, 0)=-\min _{x \in M} \psi(x)=\max _{x \in M}(-\psi(x)) .
$$

Suppose now that $d \theta \equiv 0$, then

$$
c_{0}(L)=c_{0}\left(L_{0}+\psi+\theta\right)=c_{0}\left(L_{0}+\psi\right) .
$$

Note that for all $x \in M, e+\psi(x) \geq 0$ and $L_{0} \geq 0$, therefore if $u:[0, T] \rightarrow M$ is any absolutely continuous curve we have

$$
\int_{0}^{T}\left(L_{0}(u, \dot{u})+\psi(u)+e\right) d t \geq 0
$$

which implies that $e \geq c\left(L_{0}+\psi\right) \geq c_{0}\left(L_{0}+\psi\right)$. Therefore $e=c_{0}(L)$.

We shall show now that if $\psi(x)=0$ for all $x \in M$ and $d \theta \not \equiv 0$, then $0=e<$ $c_{u}(L)$. If $d \theta \not \equiv 0$ there exists a smooth oriented embedded 2-disk $D$ in $M$ such that

$$
m \stackrel{\text { def }}{=} \int_{D} d \theta<0
$$

Fix a Riemannian metric on $M$ and let $\gamma_{\varepsilon}:\left[0, T_{\varepsilon}\right] \rightarrow M$ denote the parametrization of the boundary of $D$ (with its induced orientation) with speed $\sqrt{\varepsilon}$. The length of the boundary of $D$-which is independent of $\varepsilon$ - equals $\sqrt{\varepsilon} T_{\varepsilon} \stackrel{\text { def }}{=} l$. Now we write

$$
A_{L+\varepsilon}\left(\gamma_{\varepsilon}\right)=\int_{0}^{T_{\varepsilon}}\left(L\left(\gamma_{\varepsilon}, \dot{\gamma}_{\varepsilon}\right)+\varepsilon\right) d t=\int_{0}^{T_{\varepsilon}}\left(L_{0}\left(\gamma_{\varepsilon}, \dot{\gamma}_{\varepsilon}\right)+\varepsilon\right) d t+\int_{0}^{T_{\varepsilon}} \theta_{\gamma_{\varepsilon}}\left(\dot{\gamma}_{\varepsilon}\right) d t
$$


By Stokes Theorem we have:

$$
A_{L+\varepsilon}\left(\gamma_{\varepsilon}\right)=\int_{0}^{T_{\varepsilon}}\left(L_{0}\left(\gamma_{\varepsilon}, \dot{\gamma}_{\varepsilon}\right)+\varepsilon\right) d t+m
$$

Since $L_{0}(x, 0)=0$ and $\frac{\partial L_{0}}{\partial v}(x, 0)=0$ for all $x \in M$, there exist constants $C>0$ and $D>0$ such that for all $(x, v) \in T M$ with $\|v\| \leq D$ we have $L_{0}(x, v) \leq C\|v\|^{2}$. Therefore for $\varepsilon$ small enough we have

$$
\int_{0}^{T_{\varepsilon}}\left(L_{0}\left(\gamma_{\varepsilon}, \dot{\gamma}_{\varepsilon}\right)+\varepsilon\right) d t \leq(C \varepsilon+\varepsilon) T_{\varepsilon}=(C+1) l \sqrt{\varepsilon} .
$$

Combining the last inequality with (19) we obtain:

$$
A_{L+\varepsilon}\left(\gamma_{\varepsilon}\right) \leq(C+1) l \sqrt{\varepsilon}+m .
$$

Therefore if $\varepsilon$ is small enough, the last inequality and (18) give

$$
A_{L+\varepsilon}\left(\gamma_{\varepsilon}\right)<0
$$

which shows that $c_{u}(L)>0$ since the boundary of $D$ is a contractible curve.

As an immediate corollary of Theorem 1.3 we have,

Corollary 5.1. If $L$ is a Lagrangian of the form

$$
L(x, v)=\frac{1}{2}\langle v, v\rangle_{x}+\theta_{x}(v)
$$

with $d \theta \not \equiv 0$, then

$$
0=e<c_{u}(L) .
$$

Without the hypothesis $L(x, 0)=0$ for all $x \in M$, the second assertion of Theorem 1.3 is not true. Endow $M$ with a Riemannian metric and consider a 1-form $\theta$ with support in a neighborhood $U$ of $M$ and such that $\theta$ is not closed. Note that there exists a constant $a$ such that

$$
\frac{1}{2}\langle v, v\rangle_{x}+\theta_{x}(v)+a>0,
$$

for all $(x, v) \in T M$. Consider a smooth function $\psi: M \rightarrow \mathbf{R}$ with support in a neighborhood $V$ disjoint from $U$ and such that

$$
a=\max (-\psi) .
$$


Our Lagrangian $L$ will be

$$
L(x, v)=\frac{1}{2}\langle v, v\rangle_{x}+\theta_{x}(v)+\psi(x) .
$$

Clearly $e=a=\max (-\psi)$.

Since the supports of $\theta$ and $\psi$ are disjoint we observe that if $u:[0, T] \rightarrow M$ is any absolutely continuous curve then

$$
A_{L+e}(u) \geq 0
$$

and thus $e \geq c(L)$. It follows that for this Lagrangian

$$
e=c_{u}(L)=c_{0}(L)=c(L),
$$

but the 1-form $\theta$ is not closed.

\section{References}

[1] V. Bangert, Minimal geodesics, Ergod. Th. and Dynam. Sys. 10 (1989), 263-286.

[2] C. Camacho, A. Lins Neto, Geometric theory of foliations, Birkhäuser Boston, Inc., Boston, Mass. 1985.

[3] G. Contreras, J. Delgado, R. Iturriaga, Lagrangian flows: the dynamics of globally minimizing orbits II, preprint 1996, to appear in Bulletin of the Brazilian Math. Society.

[4] M. J. Dias Carneiro, On minimizing measures of the action of autonomous Lagrangians, Nonlinearity 8 (1995), 1077-1085.

[5] R. Mañé, Lagrangian flows: the dynamics of globally minimizing orbits, preprint 1995, International Conference on Dynamical Systems in Montevideo (a tribute to Ricardo Mañé), F. Ledrappier, J. Lewowicz, S. Newhouse (Eds.), Pitman Research Notes in Math. 362 (1996), 120-131.

[6] J. Mather, Action minimizing measures for positive definite Lagrangian systems, Math. Z. 207 (1991), 169-207.

[7] G. P. Paternain, M. Paternain, On Anosov Energy Levels of Convex Hamiltonian Systems, Math. Z. 217 (1994), 367-376.

[8] G. P. Paternain, M. Paternain, Anosov geodesic flows and twisted symplectic structures, International Conference on Dynamical Systems in Montevideo (a tribute to Ricardo Mañé), F. Ledrappier, J. Lewowicz, S. Newhouse (Eds.), Pitman Research Notes in Math. 362 (1996) 132-145.

Gabriel P. Paternain

Centro de Matemática

Facultad de Ciencias

Eduardo Acevedo 1139

Montevideo CP 11200

Uruguay

e-mail: gabriel@cmat.edu.uy
Miguel Paternain

Centro de Matemática

Facultad de Ciencias

Eduardo Acevedo 1139

Montevideo CP 11200

Uruguay

e-mail: miguel@cmat.edu.uy

(Received: October 2, 1996) 